\title{
DNS of Turbulent Lean Premixed Syngas Flames at Elevated Pressures
}

\author{
K.K.J. Ranga Dinesh, H. Shalaby, K.H. Luo, and D. Thevenin
}

\section{Introduction}

The use of low carbon content fuels as replacements or supplement for petroleum fuels offer the advantage of a much cleaner fuel with little added atmospheric carbon dioxide greenhouse burden with a low potential sulfer content. In this context, combustion of high-hydrogen content (HHC) alternative fuels with a broader reaction class such as significant quantities of hydrogen diluted with carbon monoxide is undoubtedly significant [1]. Particularly, turbulent lean premixed combustion of HHC fuels at elevated pressure is of both fundamental and practical importance for the future internal combustion engines and gas turbine engines [2].

Although various Direct Numerical Simulation (DNS) studies with simple finiterate and complex detailed chemistry provide insights into structure and dynamics of turbulent lean premixed hydrogen and hydrogen-enriched alternative fuel combustion at atmospheric pressure conditions [3-4], DNS investigations on structure and propagation of turbulent lean premixed HHC alternative fuels at elevated pressure are performed seldom in the past. For example, there is no conclusive DNS work on influence of pressure at constant turbulent Reynolds number, and influence of turbulent Reynolds number at constant pressure on flame structure and propagation for HHC lean premixed syngas flames in the thin reaction zone regime. Thus, there

K.K.J. Ranga Dinesh · H. Shalaby

Energy Technology Research Group,Faculty of Engineering and the Environment, University of Southampton, Southampton, SO17 1BJ, UK, e-mail: \{dinesh.kahanda-koralage, h. shalaby\} @soton.ac.uk

K.H. Luo

Department of Mechanical Engineering, University College London, Torrington Place, London, WC1E 7JE, UK, e-mail: k. luo@ucl.ac.uk

D. Thevenin

Laboratory of Fluid Dynamics and Technical Flows, University of Magdeburg Otto von Guericke Universitatsplatz 2, D-39106 Magdeburg, Germany, e-mail: thevenin@ovgu . de 
is a need for DNS investigation on HHC syngas turbulent lean premixed flames at different turbulent Reynolds numbers and different pressure levels.

The outwardly propagating cylindrical flame at elevated pressure is adopted as a model flame for the present investigation. In all the considered cases, twodimensional DNS were performed for HHC lean premixed hydrogen-rich hydrogencarbon monoxide syngas fuel mixture with an equivalence ratio of 0.7 . This paper will focus on answering following question: How does pressure elevation at constant turbulent Reynolds number and turbulence intensification at constant pressure couple with preferential diffusion effect on heat release rate, flame strain and curvature of lean premixed HHC syngas turbulent flame?

\section{DNS Governing Equations, Chemistry and Numerical Details}

The set of governing equations solved in DNS is the time-dependent compressible flow Navier-Stokes equations coupled with detailed chemistry and mixtureaveraged transport model via coupling with chemkin, transport and eglib. In this study an equation is solved explicitly for each and every chemical species with comprehensive detailed chemistry, simultaneously with the Navier-Stokes equations. In this investigation we have employed a hydrogen-carbon monoxide kinetic scheme developed by Goswami et al. [5]. This reaction model incorporates the thermodynamic, kinetic, and species transport properties related to elevated pressure $\mathrm{H}_{2} / \mathrm{CO}$ and oxidation, consisting of 14 species and 52 individual reactions. Furthermore, we employed the mixture-averaged transport model supplemented with a model for Soret effect (thermal diffusion) to compute the diffusive process. The full compressible governing equations together with considered thermodynamical relations, chemistry and transport models are solved using the parallel DNS flame solver, Parcomb [6]. The equations are discretised in space on a two-dimensional Cartesian grid with high-order finite difference numerical schemes. Derivatives are computed using centered explicit schemes of order six except at boundaries where the order is progressively reduced to four. Temporal integration is realized with a RungeKutta algorithm of order four.

A Courant-Friedrichs-Levy (CFL) condition for the convective terms and a Fourier condition pertaining to the diffusion terms are treated to ensure the stability of the explicit integration and determine a suitable time step. Boundary conditions are treated with the help of improved non-reflecting inflow/outflow Navier-Stokes characteristics boundary condition (NSBC) by considering additional terms in the definition of the wave amplitudes, and the relaxation treatment for the transverse gradient terms in analogy with the pressure relaxation. The initial isotropic turbulent velocity field for each case was initialised using a combined approach of digital filtering (DF)and random noise diffusion.

The square box had a length of $1.5 \mathrm{~cm}$ on each side with 1600 and $1600 \mathrm{compu}-$ tational nodes on each direction. A time step of approximately $1.8 \mathrm{~ns}$ was used for all simulations. To understand the individual effects of pressure elevation at con- 
stant turbulent Reynolds number and turbulence intensification at constant pressure on flame characteristics, six DNS test cases with the inclusion of non-unity Lewis numbers were performed by employing detailed chemical mechanism of Goswami et al.[5]. In the present study, two different pressure values of $p=2 b a r$ and $4 \mathrm{bar}$ at constant turbulent Reynolds number, and three different turbulent Reynolds number of $R e=50,100$ and 150 at constant pressure value were considered.

\section{Results and Discussion}

Figure 1 shows instantaneous images of the expanding spherical flame temperature couple with preferential diffusion at constant pressure values ranging from $\mathrm{p}=2 \mathrm{~b}$ ar to $\mathrm{p}=4 \mathrm{bar}$ under constant turbulent Reynolds number conditions ranging from $\mathrm{Re}=50$ to 150 . Two observations are made. First, it can be seen that the onset of flame cells is dominated by elevated pressure than increased turbulence. For example, it is evident from Figure 1 that pressure increase from $\mathrm{p}=2 \mathrm{bar}$ to $4 \mathrm{bar}$ at constant $\mathrm{Re}=50$ has greatly enhanced fine scale flame cells at the flame front compared to its formation due to turbulence intensification from $\mathrm{Re}=50$ to 150 at constant pressure of $\mathrm{p}=2 \mathrm{bar}$. Similar behaviour is also observed for other cases as well. Second, we observed that cellular instabilities occurring earlier for $\mathrm{p}=4 \mathrm{bar}$ compared $\mathrm{p}=2 \mathrm{bar}$.

The evolution of flame cells at elevated pressures is strongly linked with both hydrodynamic and diffusive-thermal instabilities. In particular, we observed that lean premixed HHC syngas flames tend to exhibit cellular instabilities immediately after initiation of propagation because of simultaneous excitation of the diffusive-thermal cellular instability via non-unity Lewis number. It is also believed that the onset of hydrodynamic instability is influenced by the density ratio between unburned and burned gas, and the flame thickness. These observations are consistent with previous experimental findings for appearance of hydrodynamic and diffusive-thermal cellular instabilities in outwardly expanding hydrogen/propane spherical flames at elevated pressure reported by Law et al. [7].

Figure 2 shows the scattered heat release rate and surface density function (SDF) data plotted versus progress variable at pressure levels $\mathrm{p}=2 \mathrm{bar}$ and $4 \mathrm{bar}$ under constant Re conditions, and at increased turbulence levels ranging from $\mathrm{Re}=50$ to 150 at constant pressure conditions. Several observations can be made: the scattered data of heat release rate between different pressure values and turbulent Reynolds numbers show noticeable differences highlighting the individual effects of pressure elevation at constant $\mathrm{Re}$ and turbulent intensification at constant pressure on heat release rate distributions. The scattered data of heat release rate display a large populated area at elevated pressure, $p=4 \mathrm{bar}$ and $\mathrm{Re}=50$. Also, heat release rates show large differences in its maximum value with increased pressure. Similarly, the maximum value of heat release rate is also increased with turbulence intensification at constant pressure, but the margin is lower than that observed at elevated pressure. The turbulence intensification $(\mathrm{Re}=150)$ at constant pressure also shows more populated scattered data compared to low turbulence level, $\mathrm{Re}=50$ at similar constant pressure. How- 

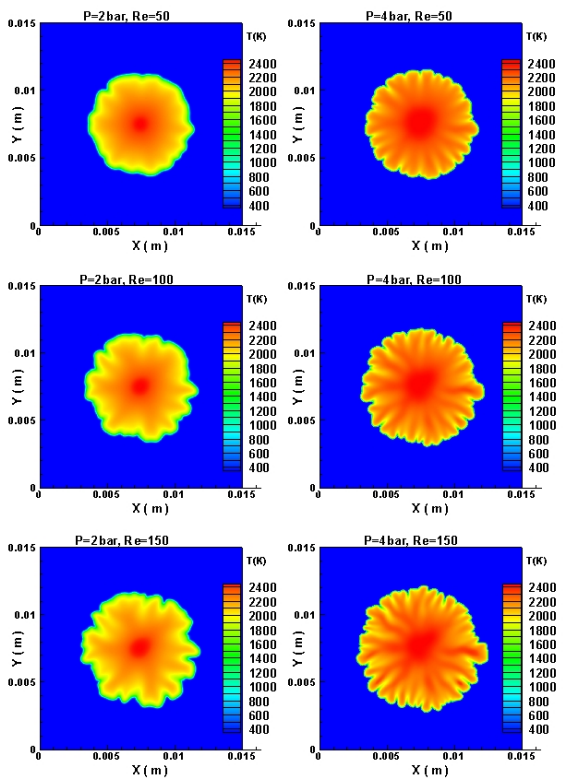

Fig. 1 Instantaneous snapshots of flame temperature at two different elevated pressure, and three different turbulent Reynolds number conditions displaying individual influence of pressure elevation at constant Reynolds number and turbulence growth at constant pressure.

ever, it is evident that turbulence intensification becomes less influential on forming widely spread population of heat release rate at elevated pressure compared to atmospheric pressure. A distinct feature of SDF across the flame indicated by Figure 2b is the appearance of slightly skewed scattered distribution with its maximum value towards the burned gas side at increased pressure and increased turbulence levels.

To understand the effect of stretch on the flame structure at different pressure levels, it is worth investigating strain rate and curvature effects on the flame front propagation. As to the analysis of strain rate and curvature effects, scattered data of strain rate and surface density function, and scattered data of curvature and surface density function are analysed. Figures $3 \mathrm{a}$ and $3 \mathrm{~b}$ show the scattered data of tangential strain rate and curvature data plotted versus SDF at different pressure levels ranging from $\mathrm{p}=2 \mathrm{bar}$ to $4 \mathrm{bar}$ under constant $\mathrm{Re}$, and at turbulence levels ranging from $R e=50$ to 150 at constant pressure.

Generally, the scattered plots of SDF and tangential strain rate show positive correlation for all cases ranging from $\mathrm{p}=2 \mathrm{bar}$ to $4 \mathrm{bar}$ and $\mathrm{Re}=50$ to 150 . It is evident that increased pressure at constant Re becomes more influential than turbulence intensification at constant pressure on generating strong positive correlation between SDF and tangential strain rate. It can be seen that the scattered data of SDF and curvature are positively correlated at negative curvature (concave). This observation is clearly apparent at elevated pressure, irrespective to the turbulence intensification. 

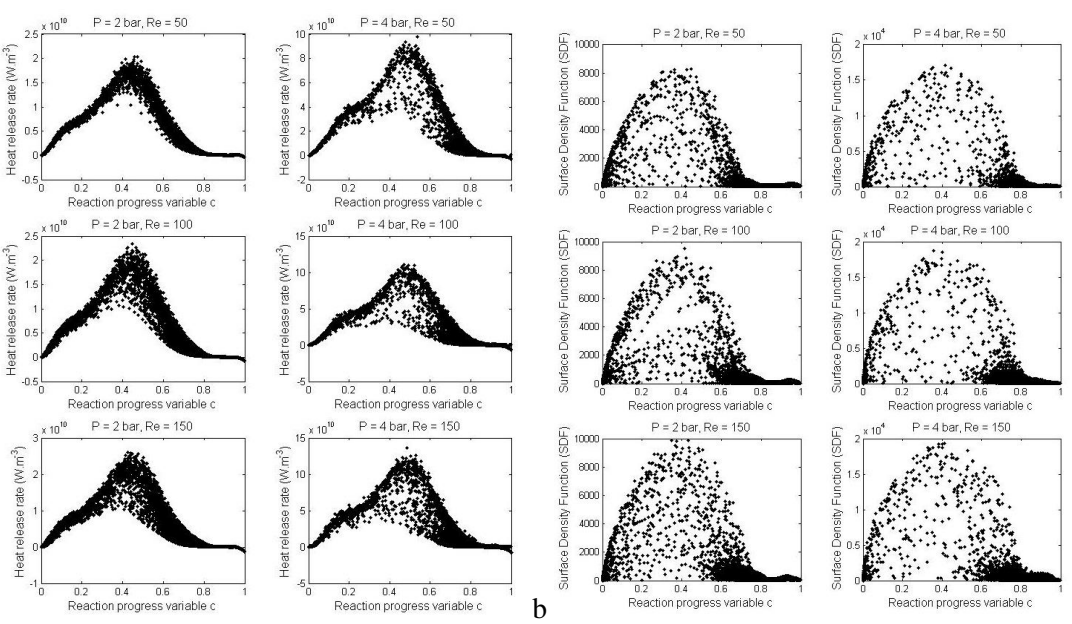

Fig. 2 Scatterplots of heat release rate (a) and surface density function (b) across the flame at two pressures varying from $\mathrm{p}=2 \mathrm{bar}$ to $4 \mathrm{bar}$ at constant $\mathrm{Re}$, and three turbulent Reynolds numbers varying from $\mathrm{Re}=50$ to 150 at constant pressure.
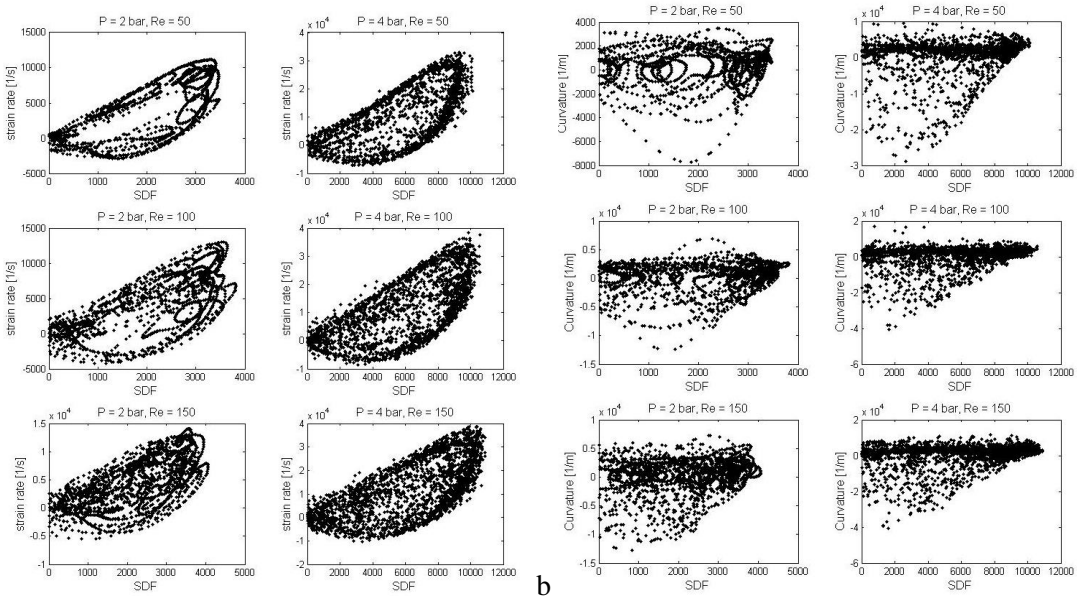

Fig. 3 Scatterplots of surface density function (SDF) with tangential strain rate (a) and curvature (b) at two pressures varying from $\mathrm{p}=2 \mathrm{bar}$ to $4 \mathrm{bar}$ at constant $\mathrm{Re}$, and three turbulent Reynolds numbers varying from $\mathrm{Re}=50$ to 150 at constant pressure. 


\section{Conclusions}

DNS of turbulent lean premixed high hydrogen content (HHC) syngas flame with detailed chemistry and multi-component transport models has been carried out for outwardly propagating cylindrical flames in the thin reaction zone regime. Under constant turbulent Reynolds number and elevated pressure conditions, highly wrinkled cellular flame structures are observed. It is found that increased pressures significantly influence distributions of hear release rate, surface density function, and correlations between surface density function and tangential strain rate, surface density function and curvature in the thin reaction zone.

\section{References}

[1]. Wall, T.F.: Combustion processes for carbon capture, Proc. Combust. Inst., 31, 31-47 (2007).

[2]. Verhelst, S. and Wallner, T.: Hydrogen-fueled internal combustion engines, Prog. Energy Combust. Sci., 35, 490-527 (2009).

[3]. Hawkes, E.R. and Chen, J.H.: Direct numerical simulation of hydrogenenriched lean premixed methane-air flames, Combust. Flame, 138, 242-258, (2004).

[4]. Aspden, A.J, Day, M.S. and Bell, J.B. : Lewis number effects in distributed flames, Proc. Combust. Inst., 33, 1473-1480 (2011).

[5]. Goswami, M, Bastiaans, R.J.M, Konnov, A.A. and de Goey, L.P.H.: Laminar burning velocity of lean hydrogen-carbon monoxide mixtures at elevated pressure, Int. J. Hydrogen Energy, 39, 1485-1498 (2014).

[6]. Hilbert, R, Tap, F, El-Rabii, H. and Thevenin, D.:Impact of detailed chemistry and transport models on turbulent combustion simulations, Prog. Energy Combust. Sci., 30, 165-193. (2004).

[7]. Law, C.K, Jomaas, G. and Bechtold, J.K.: Cellular instabilities of expanding hydrogen/propane spherical flames at elevated pressures, Proc. Combust. Inst., 30, 159-167 (2005). 